schiede des Ozonwertes würden durch eine zusätzliche dekadische Absorption von etwa 0,1 (dekadische Zenitextinktion etwa 0,01) zum Verschwinden gebracht werden. Dabei wird aber die Form der Ozonverteilungskurve nicht merklich beeinflußt, da die Absorption der zusätzlichen Schicht, wie die Messungen im Blauen bei Mondfinsternissen zeigen, in dem Schattenbereich $0 \leq \gamma^{\prime} \leq 7^{\prime}$ praktisch konstant ist.

DieWerte für die Höhe des Ozonmaximums sind für Mondfinsternisse und Ballonaufstiege in Tab. 3 zusammengestellt. Dabei ist zu bedenken, daß im Frühjahr bei den höheren Breiten das obere Maximum bei $23 \mathrm{~km}$ Höhe wegen dem zugeführten tieferen Ozon häufig nicht rein erfaßt werden kann,

\begin{tabular}{|c|c|c|c|c|}
\hline & \multicolumn{2}{|c|}{ Mondfinsternisse } & \multicolumn{2}{c|}{ Ballonaufstiege } \\
\hline & $\begin{array}{r}\text { N.od. S. } \\
\text { N.20 }\end{array}$ & $\begin{array}{c}50-60^{\circ} \\
\text { N.od. S. } \\
\text { Frühjahr }\end{array}$ & $\begin{array}{c}45^{0} \mathrm{~N} \\
\text { Sommer } \\
\text { (4 Auf- } \\
\text { stiege) }\end{array}$ & $\begin{array}{c}\text { Frühjahr } \\
\text { (6 Auf- } \\
\text { stiege) }\end{array}$ \\
\hline $\begin{array}{c}\text { Höhe des } \\
\text { Ozon- } \\
\text { maximums }\end{array}$ & 22,0 & 20,0 & 23,0 & 21,0 \\
\hline
\end{tabular}

Tab.3. Mittelwert der Höhe des Ozonmaximums. (vgl. Kurve 1 in Abb. 1) so daß sich die Maximumshöhe auf die gesamte resultierendeVerteilungskurve bezieht. Deshalb wurde auch bei einem ausgeprägten doppelten Maximum das Mittel aus beiden Höhen genommen.

Nach Tab. 3 ist die Höhe des Ozonmaximums am Äquator in guter Úbereinstimmung mit dem bei Ballonaufstieger im Sommer bei ca. $45^{0} \mathrm{~N}$ erhaltenen Wert von $23 \mathrm{~km}$. Diese Höhe entspricht der photochemischen Gleichgewichtskurve, die nach eigenen Rechnungen auch bei verschiedenen Annahmen der Sauerstoffabsorption, des Temperaturverlaufes usw. immer ein Maximum bei 22 bis $23 \mathrm{~km}$ Höhe aufweist. Im Frühjahr dagegen wird in höheren Breiten das Ozonmaximum durch das zusätzliche tiefere Ozon im Mittel um etwa $2 \mathrm{~km}$, in Übereinstimmung mit den Ballonaufstiegen, nach unten verschoben. Vielleicht kommt aber nach Tab. 3 die Höhe des Ozonmaximums bei Mondfinsternissen absolut um etwa 1 bis $2 \mathrm{~km} \mathrm{zu}$ niedrig heraus.

Diese Diskussion der vorliegenden Ozonbestimmungen bei Mondfinsternissen zeigt demnach, daß bei der Methode mit keinen wesentlichen systematischen Fehlern zu rechnen ist. Ihre Ergebnisse können daher als genügend gesichert gelten, um für das Ozonproblem herangezogen zu werden.

\title{
Die Durchtrittsüberspannung an Redoxelektroden bei mehreren aufeinanderfolgenden potentialbestimmenden Schritten
}

\author{
Von Klaus J. VetTeR \\ Aus dem Kaiser-Wilhelm-Institut für physikalische Chemie und Elektrochemie, \\ Berlin-Dahlem \\ (Z. Naturforschg. 7a, 328-333 [1952]; eingegangen am 19. Januar 1952)
}

\begin{abstract}
Es wird die Durchschnittsüberspannung für Redoxelektroden abgeleitet, die sich über mehrere aufeinanderfolgende verschiedene Elektronenübergänge einstellen. Als Ansatz wird hierfür der an einfachen Systemen verwendete Mechanismus nach Butler und Volmer verwendet. Hierbei treten mehrere Austauschstromdichten und ,Durchtrittsfaktoren" auf, deren Größe aus größeren anodischen und kathodischen Überspannungen ermittelt werden kann. Es ergeben sich verschiedene Kriterien für Elektroden mit ,„mehrfachem" potentialbestimmendem Schritt.

Der Wert des Durchtrittswiderstands am Gleichgewichtspotential wird abgeleitet. In diesen geht die Summe aller reziproken Austauschstromdichten ein. Aus der PotentialKonzentrationsabhängigkeit der Austauschstromdichten können in gleicher Weise wie beim einfachen System die potentialbestimmenden Teilschritte bestimmt und damit der Einstellungsmechanismus ermittelt werden.
\end{abstract}


$\mathrm{I}^{\mathrm{n}}$ n einer Reihe vorangehender Arbeiten ${ }^{1-8}$ über die Durchtrittsüberspannung von Redoxelektroden ist als potentialbestimmender Schritt nur eine Reaktion $\mathrm{S}_{\mathrm{o}}+\mathrm{e}^{-} \leftrightharpoons \mathrm{S}_{\mathrm{r}}$ angenommen worden, die in den einzelnen behandelten Redoxsystemen ermittelt werden konnte und die zur Deutung der Überspannung ausreichte. Auch Gerischer ${ }^{9}$ hat bei seinen Bestimmungen der Austauschstromdichte nur eine einzige derartige Größe vorausgesetzt. Ebenso ist in fast allen Arbeiten über die Wasserstoffüberspannung sehr zahlreicher Autoren nur der eine potentialbestimmende Schritt $\mathrm{H}^{+}+\mathrm{e}^{-} \leftrightharpoons \mathrm{H}$ diskutiert worden. Bei elektrochemischen Bruttoreaktionen mit einem Übergang von nur einem Elektron ist bei der bisherigen Betrachtungsweise auch kaum eine Komplikation zu erwarten.

Anders liegen die Dinge, wenn man Bruttoreaktionen mit einem Übergang von mehreren Elektronen, wie z. B. $\mathrm{Mn}^{4+}+2 \mathrm{e}^{-} \leftrightharpoons \mathrm{Mn}^{2+}$ oder $\mathrm{HNO}_{3}+$ $2 \mathrm{H}^{+}+2 \mathrm{e}^{-} \leftrightharpoons \mathrm{HNO}_{2}+\mathrm{H}_{2} \mathrm{O}$ oder $\mathrm{MnO}_{4}{ }^{\prime}+8 \mathrm{H}^{+}$ $+5 \mathrm{e}^{-} \leftrightharpoons \rightleftharpoons \mathrm{Mn}^{2+}+4 \mathrm{H}_{2} \mathrm{O}$ oder auch $2 \mathrm{H}^{+}+2 \mathrm{e}^{-}$ $\leftrightharpoons \mathrm{H}_{2}$ betrachtet. Hier müssen beide (oder alle) Elektronen nicht durch den gleichen Stoff $\mathrm{S}_{\mathrm{o}}$ aufgenommen oder vom gleichen Stoff $\mathrm{S}_{\mathrm{r}}$ an das Elektrodenmetall abgegeben werden. Es kann prinzipiell auch so sein, daß die verschiedenen Elektronen der Bruttoreaktion über verschiedene aufeinander folgende potentialbestimmende Schritte vom Metall aufgenommen bzw. abgegeben werden. Es ist dann in diesem Fall keine Dissoziations- oder Disproportionierungsfolgereaktion nötig wie bisher. Das $\mathrm{Mn}^{4+}$-Ion könnte also z. B. prinzipiell auch unter Aufnahme eines Metallelektrons in ein $\mathrm{Mn}^{3+}$-Ion und dieses unter erneuter Aufnahme eines Elektrons aus dem Metall in ein $\mathrm{Mn}^{2+}$-Ion übergehen. In diesem Fall hätte man zwei potentialbestimmende Schritte $\mathrm{Mn}^{4+}+\mathrm{e}^{-} \leftrightharpoons \mathrm{Mn}^{3+}$ und $\mathrm{Mn}^{3+}+\mathrm{e}^{-} \leftrightharpoons$ $\mathrm{Mn}^{2+}$, oder einen, zweifachen potentialbestimmenden Schritt". Tatsächlich hat jedoch dieses spezielle System nur den ,einfachen" Schritt $\mathbf{M n}^{3+}+$ $\mathrm{e}^{-} \leftrightharpoons \mathrm{Mn}^{2+}$ mit einer Disproportionierungsfolge-

1 K. J. Vetter, Z. physik. Chem. 194, 199 [1950], $\left(\mathrm{HNO}_{3} / \mathrm{HNO}_{2}\right)$.

${ }^{2}$ K. J. Vetter, Z. physik. Chem. 194, 284 [1950] $\left(i_{0}\right.$, allgemein).

${ }^{3}$ K. J. Vetter u. G. Manecke, Z. physik. Chem. 195, $270[1950]\left(\mathrm{Mn}^{3}+/ \mathrm{Mn}^{2+}\right)$.

${ }^{4}$ K. J. Vetter u. G. Manecke, 195, 337 [1950] $\left(\mathbf{M n}^{4}+/ \mathbf{M n}^{3}+\right)$.

5 K. J. Vetter, Z. physik. Chem. 196, 360 [1950] $\left(\mathrm{Ce}^{4+} / \mathrm{Ce}^{3+}\right)$.

${ }^{6}$ K. J. Vetter, Z. Elektrochem. angew. physik. Chem. 55, 121 [1951] (allgemein). reaktion, wie sich aus eigenen Polarisationsmessungen ${ }^{10,11}$ ergab. Einen solchen zweifachen potentialbestimmenden Schritt hat bereits im speziellen Bockris ${ }^{11}$ an der Wasserstoffelektrode angegeben.

Im folgenden soll nun die Durchtrittsüberspannung, der Durchtrittswiderstand und die Ermittlung der Austauschstromdichten und deren Konzentrationsabhängigkeit bei einer Redoxelektrode mit einem mehrfachen potentialbestimmenden Schritt theoretisch behandelt werden.

\section{Durchtrittsüberspannung bei einem zweifachen potentialbestimmenden Schritt}

\section{a) Allgemein}

Zur Vereinfachung sehr zahlreicher Bedingungen sei wieder ein großer Fremdelektrolytüberschuß vorausgesetzt. Hierdurch bleibt bei Variation der Konzentration der Stoffe aus der Bruttoreaktion der Aktivitätskoeffizient praktisch konstant, so daß die Aktivität proportional der Konzentration wird. Aus diesem Grunde soll im folgenden der Aktivitätskoeffizient mit in die Konstanten genommen werden, so daß mit Konzentrationen $c$ gerechnet werden kann. Das Potential $\zeta$ der diffusen Doppelschicht wird durch den Fremdelektrolytüberschuß stark herabgedrückt und praktisch von den Konzentrationen $c$ und weitgehend vom Potential $\varepsilon$ unabhängig. Der Fremdelektrolytüberschuß drückt die Überführungszahl der Ionen aus der Bruttoreaktion sehr weit herab, so daß die Ab- und Heranführung der Stoffe zur Elektrode praktisch allein durch eine voneinander unabhängige Diffusion geschieht. Ein Flüssigkeitspotential als Folge des Konzentrationsgradienten wird praktisch unterdrückt.

Als elektrochemische Bruttoreaktion für den Umsatz von 1 Faraday (l F) soll wiederum die Gleichung

$$
\begin{aligned}
m_{1} \mathrm{~S}_{1}+m_{2} \mathrm{~S}_{2}+\ldots+m_{\mathrm{p}} \mathrm{S}_{\mathrm{p}}+\mathrm{e}^{-} & \\
\quad & \leftrightharpoons\left(-m_{\mathrm{p}+1}\right) \mathrm{S}_{\mathrm{p}+1}+\ldots\left(-m_{\mathrm{q}}\right) \mathrm{S}_{\mathrm{q}}
\end{aligned}
$$

7 H. Gerischer u. K. J.Vetter, Z. physik. Chem. 197,92 [1951].

${ }^{8}$ K. J.Vetter, Z. physik. Chem. 199, 22, 285 [1952] $\left(\mathbf{J}_{2} / \mathbf{J}^{\prime}\right)$.

${ }_{9}$ H. Gerischer, Z. Elektrochem. angew. physik. Chem. a) 54, 362 [1950]; b) 54, 366 [1950]; c) 55, 98 [1951].

${ }_{10}$ J. O'M. B oc kris , Z. Elektrochem. angew. physik. Chem. 55, 105 [1951], Gl. (1).

11 Es sei definitionsgemä $B: m_{1}$ bis $m_{\mathrm{p}}>0$ und $m_{\mathrm{p}+1}$ bis $m_{\mathrm{q}}<0$ (also $-m_{\mathrm{p}+1}$ bis $-m_{\mathrm{q}}$ positiv). 
mit dem Gleichgewichtspotential nach der Nernstschen Gleichung von

$$
\varepsilon_{0}=E_{0}+\frac{R T}{F} \sum m_{\mathrm{i}} \cdot \ln c_{\mathrm{i}}
$$

verwendet werden ${ }^{11}$. Die potentialbestimmenden Schritte (zweifach) seien mit

$$
\begin{aligned}
& \mathrm{S}_{\mathrm{o}}+\mathrm{e}^{-} \leftrightharpoons \mathrm{S}_{\mathrm{m}}, \\
& \mathrm{S}_{\mathrm{m}}+\mathrm{e}^{-} \leftrightharpoons \mathrm{S}_{\mathrm{r}}
\end{aligned}
$$

definiert $^{12}$. Hierbei können die Stoffe $\mathrm{S}_{\mathrm{o}}$ und $\mathrm{S}_{\mathrm{r}}$ mit einem der Stoffe $S_{i}$ [Gl. (1)] identisch sein.

Für die Überspannung soll für jeden der beiden potentialbestimmenden Schritte Gl. (3a) und (3b) der bekannte Ansatz für die Durchtrittsüberspannung ${ }^{2}$ gemacht werden, der auf Butler ${ }^{13}$, Erdey Gruz und Volmer ${ }^{14}$ und Frumkin ${ }^{15}$ zurückgeht. Es ist also im stationären Fall

$$
\begin{aligned}
& i=k_{\mathrm{r}, \mathrm{m}} c_{\mathrm{m}} \cdot \exp \left(\frac{\alpha_{0} F}{R T} \varepsilon\right) \\
& \quad-k_{\mathrm{o}} c_{\mathrm{o}} \cdot \exp \left(-\frac{\left(1-\alpha_{\mathrm{o}}\right) F}{R T} \varepsilon\right) \\
& i=k_{\mathrm{r}} c_{\mathrm{r}} \cdot \exp \left(\frac{\alpha_{\mathrm{r}} F}{R T} \varepsilon\right) \\
&-k_{\mathrm{o}, \mathrm{m}} c_{\mathrm{m}} \cdot \exp \left(-\frac{\left(1-\alpha_{\mathrm{r}}\right) F}{R T} \varepsilon\right)
\end{aligned}
$$

$\left(c_{0}, c_{\mathrm{m}}, c_{\mathrm{r}}=\right.$ Konz. von $\mathrm{S}_{\mathrm{o}}, \mathrm{S}_{\mathrm{m}}, \mathrm{S}_{\mathrm{r}} ; \varepsilon=$ Potential; $i=$ Polarisationsstromdichte, anod. $i>0$, kath. $i<0$;

$k_{0}, k_{\mathrm{m}}, k_{\mathrm{r}}=$ Geschwindigkeitskonstanten

$[\mathrm{Amp} \cdot \mathrm{cm} / \mathrm{Mol}]^{16}$.

$\alpha_{0}$ und $\alpha_{\mathrm{r}}$ sind hierin die ,Durchtrittsfaktoren“ der Reaktionen (3a) bzw. (3b). Sie können trotz des gleichen Elektrolyten und Elektrodenmetalls verschieden sein. Am Gleichgewichtspotential $\varepsilon_{0}$ ist $i=0$ und somit die Austauschstromdichten $i_{0, o}$ und $i_{0, \mathrm{r}}$ der beiden potentialbestimmenden Schritte Gl. $(3 \mathrm{a}, \mathrm{b})$

$$
\begin{aligned}
& i_{0, \mathrm{o}}=k_{\mathrm{r}, \mathrm{m}} c_{\mathrm{m}} \cdot \exp \left(\frac{\alpha_{\mathrm{o}} F}{R T} \varepsilon_{0}\right) \\
&=k_{\mathrm{o}} \mathrm{c}_{\mathrm{o}} \cdot \exp \left(-\frac{\left(1-\alpha_{\mathrm{o}}\right) F}{R T} \varepsilon_{0}\right),
\end{aligned}
$$

12 Der Index o soll sich auf den ,oxydierten“ und Index $\mathrm{r}$ auf den ,reduzierten" Stoff beziehen. $\mathrm{m}$ bezieht sich auf den dazwischen liegenden Stoff im „mittleren“ Oxydations- bzw. Reduktionszustand.

${ }_{13}$ J. A. V. Butler, Trans. Faraday Soc. 19, 734 [1924]; 28, 379 [1932].

14 T. Erdey - Gruz, u. M.Volmer, Z. physik. Chem. Abt. A 150, 203 [1930].

$$
\begin{aligned}
& i_{0, \mathrm{r}}=k_{\mathrm{r}} c_{\mathrm{r}} \cdot \exp \left(\frac{\alpha_{\mathrm{r}} F}{R T} \varepsilon_{0}\right) \\
&=k_{0, \mathrm{~m}} c_{\mathrm{m}} \cdot \exp \left(-\frac{\left(1-\alpha_{\mathrm{r}}\right) F}{R T} \varepsilon_{0}\right) .
\end{aligned}
$$

Nach Elimination von $c_{\mathrm{m}}$ aus Gl. $(4 \mathrm{a}, \mathrm{b})$ und Einsetzen der Ausdrücke für $i_{0, \text { o }}$ und $i_{0, \mathrm{r}}$ Gl. $(5 \mathrm{a}, \mathrm{b})$ ergibt sich unter Berücksichtigung der Definition der Überspannung $\eta=\varepsilon-\varepsilon_{0}$ für die Stromdichte als Funktion von $\eta$

$$
i=\frac{\exp \left(\frac{\alpha_{0}+\alpha_{\mathrm{r}}}{R T} F \eta\right)-\exp \left(-\frac{2-\left(\alpha_{0}+\alpha_{\mathrm{r}}\right)}{R T} F \eta\right)}{\frac{1}{i_{0, \mathrm{r}}} \cdot \exp \left(\frac{\alpha_{0} F}{R T} \eta\right)+\frac{1}{i_{0,0}} \cdot \exp \left(-\frac{\left(1-\alpha_{\mathrm{r}}\right) F}{R T} \eta\right)} .
$$

Voraussetzung für die Gültigkeit dieser Gl. (6) ist, daß die Konzentration $c_{\mathrm{m}}$ so klein bleibt, daß eine Abdiffusion von $\mathrm{S}_{\mathrm{m}} \mathrm{zu}$ vernachlässigen ist.

\section{b) Stromdichte-Potentialkurve bei großen}

\section{Überspannungen}

Für große positive wie negative Überspannungen vereinfacht sich Gl. (6), wie es auch beim einfachen potentialbestimmenden Schritt beobachtet wird, sehr wesentlich. Bei genügend großer anodischer Überspannung $\eta>0$ (also auch $i>0$ ) geht Gl. (6) in Gl. (7a)

$$
i=i_{0, \mathrm{r}} \cdot \exp \left(\frac{\alpha_{\mathrm{r}} F}{R T} \eta\right)=k_{\mathrm{r}} c_{\mathrm{r}} \cdot \exp \left(\frac{\alpha_{\mathrm{r}} F}{R T} \varepsilon\right)
$$

über. Für genügend große kathodische Überspannungen $(\eta<0 ; i<0)$ wird

$$
\begin{aligned}
i=-i_{0,0} & \cdot \exp \left(-\frac{\left(1-\alpha_{0}\right) F}{R T} \eta\right) \\
& =-k_{\mathrm{o}} c_{\mathrm{o}} \cdot \exp \left(-\frac{\left(1-\alpha_{0}\right) F}{R T} \varepsilon\right) .
\end{aligned}
$$

In beiden Fällen ergịt sich eine lineare Abhängigkeit des Potentials $\varepsilon$ (Überspannung $\eta$ ) vom $\log i$, so wie es bei Polarisationsmessungen vielfach beobachtet wird. Hierbei hängt die anodische Überspannung nur vom potentialbestimmenden Teilschritt auf der reduzierten Seite (Index r) und die kathodische von dem auf der oxydierten Seite (In$\operatorname{dex} 0) a b$.

15 A. Frumkin, Z. physik. Chem. Abt. A 164, 121 [1933].

${ }^{16}$ In $k_{0}, k_{\mathrm{m}}$ und $k_{\mathrm{r}}$ soll schon der Einfluß der Konzentrationsverschiebung in der diffusen Doppelschicht $c^{\prime}=c \cdot \exp (z F \zeta / R T)$ mit $z=$ Ionenladung unter der Voraussetzung enthalten sein, daß sich $\zeta$ im betrachteten Potentialbereich wegen seiner Kleinheit infolge des Fremdelektrolytüberschusses nur wenig ändert. 
Aus der Neigung von $\eta=f(\log i)$ ergibt sich in bekannterWeise der „,Durchtrittsfaktor" $\alpha$. Aus der anodischen Überspannung folgt hierbei $\alpha_{\mathrm{r}}$ und aus der kathodischen $\alpha_{0}$. Da im allgemeinen $\alpha_{0} \neq \alpha_{\mathrm{r}}$ sein wird, so hat man bei einer Abweichung der anodisch und kathodisch erhaltenen $\alpha$-Werte ein Kriterium für das Auftreten eines mehrfachen (zweifach) potentialbestimmenden Schrittes.

Aber dies ist nicht das einzige Kriterium. Die Verlängerung des linearen Verlaufs der logarithmischen Stromspannungskurve bis auf das Gleichgewichtspotential $\varepsilon_{0}(\eta=0)$ ergibt die Austauschstromdichte $i_{0, \mathrm{r}}$ bzw. $i_{0,0}$, je nachdem ob es sich um die anodische bzw. kathodische Polarisation handelt. Eine Abweichung der anodisch und kathodisch ermittelten Austauschstromdichten ist somit ebenfalls ein weiteres Kriterium für einen mehrfachen (zweifachen) potentialbestimmenden Schritt.

Es besteht somit die Möglichkeit, aus den großen Überspannungen [nach Gl. $(7 \dot{a}, b)$ ] die für die gesamte Polarisation nach Gl. (6) maßgebenden vier Größen $\alpha_{0}, \alpha_{\mathrm{r}}, i_{0, o}$ und $i_{0, \mathrm{r}} \mathrm{zu}$ bestimmen, womit nach Gl. (6) auch eine Berechnung für kleine Überspannungen möglich sein muß.

c) Ein potentialbestimmender Schritt ist praktisch ungehemmt

Für den Fall, daß die eine Austauschstromdichte um mehrere Größenordnungen größer als die andere ist, wird experimentell ein Erreichen eines durch Gl. (7 a) oder (7 b) darzustellenden Stromspannungsverlaufs nicht mehr möglich sein. Es soll hier der Grenzfall behandelt werden, der eintritt, wenn eine der beiden Austauschstromdichten unendlich groß wird, d.h. also, daß ein potentialbestimmender Schritt ungehemmt ist. Für diesen Fall muß sich also bei allen Polarisationspotentialen $\varepsilon$ das Konzentrationsverhältnis $c_{\mathrm{o}} / c_{\mathrm{m}}$ bzw. $c_{\mathrm{m}} / c_{\mathrm{r}}$ einstellen, bei dem $\varepsilon$ gleich dem Gleichgewichtspotential $\varepsilon_{0}^{\prime}$ für diesen Einzelvorgang ist.

Im Falle, daß der Vorgang o, Gl. (3 a) ungehemmt ist, also $i_{0,0}=\infty$ ist, ergibt sich aus Gl. (6) für die gesamte Stromdichte-Potentialkurve

$i=i_{0, \mathrm{r}} \cdot\left[\exp \left(\frac{\alpha_{\mathrm{r}} F}{R T} \eta\right)-\exp \left(-\frac{\left(2-\alpha_{\mathrm{r}}\right) F}{R T} \eta\right)\right]$

$$
\text { bei } i_{0, o}=\infty . \quad(8 \mathrm{a})
$$

Ist dagegen derVorgang r, Gl. (3 b), vollständig ungehemmt, also $i_{0, \mathrm{r}}=\infty$, so ist entsprechend $i=i_{0,0} \cdot\left[\exp \left(\frac{\left(1+\alpha_{0}\right) F}{R T} \eta\right)-\exp \left(-\frac{\left(1-\alpha_{0}\right) F}{R T} \eta\right)\right]$ bei $i_{0, \mathrm{r}}=\infty$.

Die lineare Abhängigkeit des Potentials $\varepsilon$ oder der Überspannung $\eta$ vom $\log i$ ist sowohl für große anodische wie auch für große kathodische $\eta$-Werte erfüllt. Die lineare Verlängerung dieser Geraden bis auf das Gleichgewichtspotential $\varepsilon_{0}(\eta=0)$ führt hier jedoch auf den gleichen Stromdichtewert, der Austauschstromdichte des gehemmten Vorganges. Hierin unterscheidet sich das Verhalten der Überspannung also nicht von dem bei einem einfachen potentialbestimmenden Schnitt. Jedoch besteht ein Unterschied in der Größe der ,,effektiven“" Durchtrittsfaktoren. Die aus den Neigungen der Geraden zu berechnenden Faktoren addieren sich im vorliegenden zweifachen Schritt zu 2, während ihre Summe beim einfachen Schritt nur 1 ist. Also auch bei völliger Enthemmung der Schritte bis auf einen erhält man aus der Summe der Neigungen bei großen Überspannungen die Vielfachheit des Schrittes. Auffallend ist hierbei, daß Neigungen mit einem effektiven Faktor größer als eins im vorliegenden Fall herauskommen.

\section{Stationärer Durchtrittswiderstand $\mathbf{R}_{\mathbf{D}}$}

Der stationäre Durchtrittswiderstand $R_{\mathrm{D}}$ oder auch der Widerstand der Durchtrittsüberspannung am Gleichgewichtspotential $\varepsilon_{0}$ ist $^{6}$

$$
R_{\mathrm{D}}=\left(\frac{\partial \varepsilon}{\partial i}\right)_{i=0}=\left(\frac{\partial \eta}{\partial i}\right)_{i=0}
$$

bei Konstanz aller Konzentrationen bis auf $c_{\mathrm{m}}$. Für einen einfachen Schritt ist $R_{\mathrm{D}}=R T / F \cdot i_{0}\left(i_{0}=\right.$ Austauschstromdichte $^{2,9 a}$ ). Für den zweifachen Schritt ergibt sich ein entsprechender Ausdruck durch Differentiation von Gl. (6) nach $\eta$ (bzw. $\varepsilon$ ). Bei nachträglichem Einsetzen von $\eta=0$, also $\varepsilon=\varepsilon_{0}$ und $i=0$, wird zunächst

$$
\left(\frac{\partial i}{\partial \eta}\right)_{\eta=0}=\frac{\left(\frac{1}{i_{0, \mathrm{r}}}+\frac{1}{i_{0, \mathrm{o}}}\right) \cdot \frac{2 F}{R T}-0}{\left(\frac{1}{i_{0, \mathrm{r}}}+\frac{1}{i_{0, \mathrm{o}}}\right)^{2}}
$$

und schließlich

$$
\begin{aligned}
R_{\mathrm{D}}=\left(\frac{\partial \eta}{\partial i}\right)_{i=0}=\left(\frac{\partial \varepsilon}{\partial i}\right)_{i=0} & \\
& =\frac{R T}{2 F} \cdot\left(\frac{1}{i_{0,0}}+\frac{1}{i_{0, \mathrm{r}}}\right) .
\end{aligned}
$$


Ist hier wieder ein Schritt völlig ungehemmt, so folgt ein Ausdruck, der sich von dem mit einfachem Schritt um den Faktor 2 unterscheidet. Dieser Faktor hängt mit dem von Bockris ${ }^{10} \mathrm{im}$ Zusammenhang mit der Wasserstoffüberspannung definierten stöchiometrischen Faktor $\mu=2 F i_{0} R_{\mathrm{D}} / R T$ zusammen, der somit für einen einfachen Schritt $\mu=2$ und für einen zweifachen Schritt $\mu=1$ ist, wenn der eine Teilschritt ungehemmt ist.

\section{Durchtrittswiderstand bei mehrfach potentialbestimmenden Schritten}

Es sei jetzt noch kurz eine Erweiterung der Gl. (10) auf Elektroden mit mehr als zweifachem Schritte durchgeführt. Liegt der folgende $n$-fache potentialbestimmende Schritt

$$
\begin{array}{ll}
\mathrm{S}_{0}+\mathrm{e}^{-} \leftrightharpoons \mathrm{S}_{1} & i_{0,1} \\
\mathrm{~S}_{1}+\mathrm{e}^{-} \mathrm{S}_{2} & i_{0,2} \\
\ldots \ldots \ldots & \ldots . \\
\mathrm{S}_{\mathrm{n}-1}+\mathrm{e}^{-} \leftrightharpoons \mathrm{S}_{\mathrm{n}} & i_{0, \mathrm{n}}
\end{array}
$$

vor, so ist der Durchtrittswiderstand $R_{\mathrm{D}}$, wie der noch folgende Induktionsbeweis zeigen wird

$$
R_{\mathrm{D}}=\left(\frac{\partial \eta}{\partial i}\right)_{i=0}=\frac{R T}{n F} \sum_{1}^{n} v \frac{1}{i_{0, \nu}} .
$$

Zum Beweis soll Gl. (12) als richtig vorausgesetzt und der Ausdruck für $R_{\mathrm{D}}$ hèrgeleitet werden, wenn an die Reaktion Gl. (11) noch der $(\mathrm{n}+1)$-te Schritt

$$
\mathrm{S}_{\mathrm{n}}+\mathrm{e}^{-} \leftrightharpoons \mathrm{S}_{\mathrm{r}}\left(=\mathrm{S}_{\mathrm{n}+1}\right)
$$

angehängt wird. Da die Diffusionsüberspannung voraussetzungsgemäß null sein soll, müssen die Konzentrationen $c_{\mathrm{o}}$ und $c_{\mathrm{r}}$ unabhängig von $i$ sein. Die Zwischenkonzentrationen $c_{1}$ bis $c_{\mathrm{n}-1}$ bzw. $c_{\mathrm{n}}$ müssen dagegen von $i$ abhängen, wenn die Austauschstromdichten $i_{0, \mathrm{r}}$ nicht alle untereinander gleich sind. Wird somit Reaktion Gl. (13) an Reaktion Gl. (11) angehängt, so wird jetzt $c_{\mathrm{n}}$ mit $i$ variabel, dagegen $c_{\mathrm{n}+1}=c_{\mathrm{r}}$ bleibt konstant. Für die Größe von $R_{\mathrm{D}, \mathrm{n}+1}=(\partial \eta / \partial i)_{\mathrm{n}+1}$ kann unter Berücksichtigung der Stromabhängigkeit von $c_{\mathrm{n}}$ entweder aus Reaktion Gl. (11) oder aus Reaktion Gl. (13) eine Beziehung abgeleitet werden. Aus beiden Gleichungen ist dann $c_{\mathrm{n}} \mathrm{zu}$ eliminieren.

Der gesamte Polarisationswiderstand $R_{\mathrm{p}}$ am Gleichgewichtspotential setzt sich additiv aus den Anteilen $R_{\mathrm{D}}$ des Durchtritts- und $R_{\mathrm{k}}$ der Konzentrationsüberspannung zusammen, also $R_{\mathrm{p}}=R_{\mathrm{D}}+$ $R_{\mathrm{k}}$. $R_{\mathrm{D}}$ wird durch den vorausgesetzten Ausdruck
Gl. (12) gegeben. $R_{\mathrm{k}}$ ist auf Grund der NernstschenGleichung $\left(\mathrm{S}_{\mathrm{o}}\right.$ und $\mathrm{S}_{\mathrm{r}}$ Anfangs- und Endstoff des gesamten mehrfachen Schrittes in allgemeiner Betrachtung)

$$
\begin{gathered}
\varepsilon_{0}=E_{0}+\frac{R T}{n F} \cdot \ln \frac{c_{\mathrm{o}}}{c_{\mathrm{r}}} \\
R_{\mathrm{k}}=\left(\frac{\partial \varepsilon}{\partial i}\right)_{i=0}=\left(\frac{\partial \eta}{\partial i}\right)_{i=0} \\
=\frac{R T}{n F}\left(\frac{1}{c_{\mathrm{o}}} \frac{\partial c_{\mathrm{o}}}{\partial i}-\frac{1}{c_{\mathrm{r}}} \frac{\partial c_{\mathrm{r}}}{\partial i}\right) .
\end{gathered}
$$

Auf die Reaktion Gl.(11) angewandt, ergibt sich für

$$
\begin{aligned}
R_{\mathrm{p}}=R_{\mathrm{D}}+R_{\mathrm{k}} & =\frac{R T}{n F} \sum_{1}^{n} \frac{1}{i_{0, v}}-\frac{R T}{n F} \frac{1}{c_{\mathrm{n}}} \frac{\partial c_{\mathrm{n}}}{\partial i} \\
& =R_{\mathrm{D}, \mathrm{n}+1},
\end{aligned}
$$

und unter Anwendung auf die Reaktion Gl.(13)

$$
\begin{aligned}
R_{\mathrm{D}}+R_{\mathrm{k}} & =\frac{R T}{F} \frac{1}{i_{0, \mathrm{n}+1}}+\frac{R T}{F} \frac{1}{c_{\mathrm{n}}} \frac{\partial c_{\mathrm{n}}}{\partial i} \\
& =R_{\mathrm{D}, \mathrm{n}+1} .
\end{aligned}
$$

Aus Gl. $(16 \mathrm{a}, \mathrm{b})$ folgt für $R_{\mathrm{D}, \mathrm{n}+1}$ nach Elimination von $\partial c_{n} / \partial i$

$$
R_{\mathrm{D}, \mathrm{n}+1}=\left(\frac{\partial \eta}{\partial i}\right)_{i=1}=\frac{R T}{(n+1) F} \sum_{1}^{\mathrm{n}+1} \frac{1}{i_{0, v}}
$$

die Gl. (12) für $n+1$. Da Gl. (12) auch für $n=1$ (und sogar auch für $n=2$ ) anderweitig hergeleitet wurde, ist somit vom theoretisch-mathematischen Standpunkt aus ihre Richtigkeit bewiesen.

\section{Potentialabhängigkeit der Austauschstromdichten bei Variation einer Konzentration}

a) Einzelaustauschstromdichten $i_{0,0}$ und $i_{0, \mathrm{r}}$

Wenn in einem Redoxsystem die Konzentration $c_{\mathrm{i}}$ eines Stoffes $\mathrm{S}_{\mathrm{i}}$ geändert wird, so ändert sich hierbei das Gleichgewichtspotential $\varepsilon_{0}$ und die Austauschstromdichte $i_{0}$. Es kann daher $i_{0}$ in Beziehung zu $\varepsilon_{0}$ bei Variation dieser Konzentration $c_{\mathrm{i}}$ und Konstanthalten aller anderen $c_{v \neq \mathrm{i}}$ gesetzt werden. Hierbei hat sich bei Elektroden mit einfachem potentialbestimmenden Schritt eine einfache lineare Beziehung zwischen $\ln i_{0}$ und $\varepsilon_{0}$ ergeben ${ }^{3,6}$. Es war dort also $\partial \ln i_{0} / \partial \varepsilon_{0}$ konstant.

Eine sehr ähnliche einfache Beziehung folgt auch hier. Nach Logarithmieren von Gl. (5) und Differentiation nach dem Gleichgewichtspotential ergibt sich 


$$
\begin{aligned}
& \left(\frac{\partial \ln i_{0, \mathrm{o}}}{\partial \varepsilon_{0}}\right)_{c_{\nu \pm \mathrm{i}}}=\left(\frac{\partial \ln c_{0}}{\partial \varepsilon_{0}}\right)_{c_{\nu \pm \mathrm{i}}}-\frac{\left(1-\alpha_{0}\right) F}{R T}, \\
& \left(\frac{\partial \ln i_{0, \mathrm{r}}}{\partial \varepsilon_{0}}\right)_{c_{\nu \pm \mathrm{i}}}=\left(\frac{\partial \ln c_{\mathrm{r}}}{\partial \varepsilon_{0}}\right)_{c_{\nu \pm \mathrm{i}}}+\frac{\alpha_{\mathrm{r}} F}{R T} .
\end{aligned}
$$

Der Stoff $\mathrm{S}_{\mathrm{o}}$ bzw. $\mathrm{S}_{\mathrm{r}}$ steht nun nach dem Massenwirkungsgesetz entsprechend den stöchiometrischen Faktoren $z_{\nu, o}$ bzw. $z_{\nu, \mathrm{r}}$ mit den Stoffen $\mathrm{S}_{\nu}$ der elektrochemischen Bruttoreaktion im Gleichgewicht ${ }^{17}$, so daß gilt

$$
c_{\mathrm{o}}=K_{\mathrm{o}} \cdot \Pi c_{\nu}^{z, o} \quad \text { bzw. } c_{\mathrm{r}}=K_{\mathrm{r}} \cdot \Pi c_{\nu}^{z, \mathrm{r}} \text {. }
$$

Es ist somit nach Gl. (19) und der Nernstschen Gleichung (2)

$$
\left(\frac{\partial \ln c_{\mathrm{o}}}{\partial \varepsilon_{0}}\right)_{c_{\nu \ddagger \mathrm{i}}}=\frac{\partial \ln c_{0}}{\partial \ln c_{\mathrm{i}}} \frac{\partial \ln c_{\mathrm{i}}}{\partial \varepsilon_{0}}=\frac{z_{\mathrm{i}, o}}{m_{\mathrm{i}}} \frac{F}{R T}
$$

und entsprechend

$$
\left(\frac{\partial \ln c_{\mathrm{r}}}{\partial \varepsilon_{0}}\right)_{c_{\nu_{ \pm} \mathrm{i}}}=\frac{\partial \ln c_{\mathrm{r}}}{\partial \ln c_{\mathrm{i}}} \frac{\partial \ln c_{\mathrm{i}}}{\partial \varepsilon_{0}}=\frac{z_{\mathrm{i}, \mathrm{r}}}{m_{\mathrm{i}}} \frac{F}{R T} .
$$

Außerdem besteht zwischen den beiden Differentialquotienten der Gl. $(20 \mathrm{a}, \mathrm{b})$ bei einem zweifachen Schritt die Beziehung

$$
\frac{\partial \ln c_{0}}{\partial \varepsilon_{0}}-\frac{\partial \ln c_{\mathrm{r}}}{\partial \varepsilon_{0}}=2 \frac{F}{R T},
$$

da $\varepsilon_{0}=E_{0}+\frac{R T}{2 F} \cdot \ln \frac{c_{\mathrm{o}}}{c_{\mathrm{r}}}$ ist. Es ergibt sich somit für die Potential-Konzentrationsabhängigkeit der beiden Austauschstromdichten $i_{0, o}$ und $i_{0, \mathrm{r}}$ aus Gl: $(18 \mathrm{a}, \mathrm{b}),(20 \mathrm{a}, \mathrm{b})$ und $(2 \mathrm{l})$

$$
\begin{aligned}
\left.\frac{\partial \ln i_{0, \mathrm{o}}}{\partial \varepsilon_{0}}\right)_{c_{\nu \neq \mathrm{i}}} & =\frac{F}{R T}\left(\alpha_{\mathrm{o}}+\frac{z_{\mathrm{i}, \mathrm{o}}}{m_{\mathrm{i}}}-1\right) \\
& =\frac{F}{R T}\left(\alpha_{\mathrm{o}}+\frac{z_{\mathrm{i}, \mathrm{r}}}{m_{\mathrm{i}}}+1\right),
\end{aligned}
$$

$$
\begin{aligned}
\left(\frac{\partial \ln i_{0, \mathrm{r}}}{\partial \varepsilon_{0}}\right)_{c_{\nu \neq \mathrm{i}}}= & \frac{F}{R T}\left(\alpha_{\mathrm{r}}+\frac{z_{\mathrm{i}, \mathrm{r}}}{m_{\mathrm{i}}}\right) \\
& =\frac{F}{R T}\left(\alpha_{\mathrm{r}}+\frac{z_{\mathrm{i}, \mathrm{o}}}{m_{\mathrm{i}}}-2\right) .
\end{aligned}
$$

Da die Differentialquotienten unabhängig vom Gleichgewichtspotential $\varepsilon_{0}$ sind, so muß auch hier eine lineare Abhängigkeit zwischen $\ln i_{0}$ und $\varepsilon_{0}$ bestehen, aus deren Neigungswert Gl. (22) auf die Größe von $z_{\mathrm{i}, o}$ bzw. $z_{\mathrm{i}, \mathrm{r}}$ geschlossen werden kann, da $m_{\mathrm{i}}$ aus der Bruttoreaktionsgleichung und $\alpha_{\mathrm{o}}$ bzw. $\alpha_{\mathrm{r}}$ aus der Stromdichte-Potentialkurve bekannt sind. Bei Kenntnis von $z_{\mathrm{i}, o}$ bzw. $z_{\mathrm{i}, \mathrm{r}}$, d. h. bei Kenntnis der Stöchiometrie zwischen den Stoffen $S_{i}$ und $S_{0}$ bzw. $S_{r}$ werden mit der gleichen Sicherheit, wie in der chemischen Reaktionskinetik, die Stoffe $S_{0}$ und $\mathrm{S}_{\mathrm{r}}$ und damit die potentialbestimmenden Schritte zu ermitteln sein.

\section{b) ,Scheinbare“ Austauschstromdichte $i_{0}^{*}$}

Aus dem Durchtrittswiderstand $R_{\mathrm{D}}$ am Gleichgewichtspotential ist zunächst nach $i_{0}^{*}=R T / 2 F R_{\mathrm{D}}$ [vgl. Gl. (10)] nur eine ,scheinbare" Austauschstromdichte $i_{0}^{*} \mathrm{zu}$ ermitteln, die nach

$$
\frac{1}{i_{0}^{*}}=\frac{1}{i_{0, \mathrm{o}}}+\frac{1}{i_{0, \mathrm{r}}}
$$

mit $i_{0, o}$ und $i_{0, \mathrm{r}}$ in Beziehung steht. Es interessiert daher auch noch die Potential-Konzentrationsabhängigkeit dieser Größe $i_{0}^{*}$, da diese direkt der Messung zugänglich ist. Sie ergibt sich aus Gl.(22) und (23) unter Anwendung der Regeln der Differentation $\mathrm{zu}$

$$
\left(\frac{\partial \ln i_{0}^{*}}{\partial \varepsilon_{0}}\right)_{c_{\nu \neq \mathrm{i}}}=\frac{F}{R T}\left[\frac{z_{\mathrm{i}, \mathrm{o}}}{m_{\mathrm{i}}}-\frac{2-\alpha_{\mathrm{r}}+\left(1-\alpha_{0}\right) \cdot \exp \left(\frac{\alpha_{\mathrm{r}}-\alpha_{0}-1}{R T} F\left(\varepsilon_{0}-\varepsilon_{0}^{\prime}\right)\right)}{1+\exp \left(\frac{\alpha_{\mathrm{r}}-\alpha_{0}-1}{R T} F\left(\varepsilon_{0}-\varepsilon_{0}^{\prime}\right)\right)}\right]
$$

Hierin ist $\varepsilon_{0}^{\prime}$ das Gleichgewichtspotential, bei dem $i_{0, \mathrm{o}}=i_{0, \mathrm{r}}$ ist. Für größere positive bzw. negative $\left(\varepsilon_{0}-\varepsilon_{0}^{\prime}\right)$-Werte geht Gl. (24) wie zu fordern, in die Gl. (22a) bzw. (22 b) über.

Aus den angegebenen Kriterien ist es also nicht nur möglich, die Existenz eines zwei- oder mehrsein. fachen potentialbestimmenden Schrittes an einer Elektrode zu erkennen, sondern es lassen sich auch in der gleichen Einfachheit wie beim einfachen Schritt die potentialbestimmenden Mechanismen ermitteln und damit der Einstellungsmechanismus bestimmen.

Für wertvolle Diskussionen möchte ich Herrn Professor K. F. Bonhoeffer vielmals danken. 